\title{
OPEN Lipidomic response of the entomopathogenic fungus Beauveria bassiana to pyrethroids
}

\author{
Anna Litwin $\mathbb{D}^{\mathbb{D}}$, Przemysław Bernat ${ }^{\mathbb{D}}$, Monika Nowak $\mathbb{D}^{\mathrm{D}}$, Mirosława Słaba $\mathbb{D}$ \& \\ Sylwia Różalska®D
}

Pyrethroids are chemical insecticides that are widely used to control pests. Entomopathogenic fungi are considered environmentally safe alternatives to these compounds. Pyrethroids and entomopathogenic fungi not only co-exist in the environment but can also be applied together in pest control. They are often found in contact with each other, and thus, it seems important to understand their interactions at the cellular level. In this study, we analyzed whether pyrethroids could influence the phospholipid profile of Beauveria bassiana and whether membrane changes are one of the mechanisms by which these fungi adapt to unfavorable environmental conditions. The results of our study revealed that pyrethroids changed the phospholipid profile and increased the cell membrane permeability of $B$. bassiana, which enabled them to enter and accumulate within the fungal cells, resulting in oxidative stress. Pyrethroids influenced the amount of neutral lipids, caused a decrease in sodium content, and also temporarily lowered the level of the secondary metabolite oosporein in the studied fungi. These findings indicate that the effect of pyrethroids on entomopathogenic fungi may be more complex than originally thought and that lipidomic studies can aid in fully understanding the influence of these chemicals on the mentioned group of fungi.

Pyrethroids are insecticides used to control arthropods in agriculture, forestry, and greenhouses. They are neurotoxic substances that disrupt the nervous system of insects by affecting the activity of sodium and chloride channels ${ }^{1-3}$. Due to the widespread use as well as the lipophilic and hydrophobic properties of pyrethroids, their residues can be found in the environment (e.g., soil, surface water, and sediments) ${ }^{2,4}$. Despite the fact that pyrethroids are less harmful and safer than other insecticides, it has been proven that they exert negative effects on living organisms ${ }^{4}$. Pyrethroids have rather long half-lives in soil and are also poorly soluble in water due to their strongly hydrophobic nature ${ }^{2}$.

The soil is the habitat of many microorganisms, including entomopathogenic fungi, which are parasites controlling the density of insect populations in nature. It has been estimated that the propagules of Ascomycota entomopathogenic fungi could reach $10^{6}$ per g of soil ${ }^{5}$. Entomopathogens of the genera Beauveria and Metarhizium are widely used as bioinsecticides and introduced into the environment to protect crops ${ }^{6}$. Due to their longterm action, these fungi can be used together with chemical insecticides or as their alternatives in pest control ${ }^{7}$.

Entomopathogenic fungi and pyrethroids can not only be applied together to control pests but also coexist in the environment, and are therefore often found in contact with each other. Thus, it seems important to understand their interactions at the cellular level.

So far, only a few studies have analyzed the response of entomopathogenic fungi to pyrethroids at the cellular level, with most of them focusing only on fungal growth in the presence of these compounds ${ }^{8-11}$. Pyrethroids do not mostly adversely affect fungal growth ${ }^{12}$, and hence are considered to not cause any significant cell damage. Forlani et al. ${ }^{8}$ revealed that exposure of Beauveria bassiana to the pyrethroid deltamethrin led to the induction of two cytochrome P450 genes and antioxidant system genes such as superoxide dismutase, catalase, and glutathione $\mathrm{S}$-transferase. These findings suggest that the defense mechanisms of $B$. bassiana are activated in response to the harmful effects of deltamethrin.

In this study, we analyzed whether pyrethroids, which are considered nontoxic to entomopathogenic fungi, influenced the phospholipid profile of B. bassiana due to the fact that changes in the membrane structure and properties are one of the mechanisms that allow microorganisms to adapt to unfavorable environmental

Department of Industrial Microbiology and Biotechnology, Institute of Microbiology, Biotechnology and Immunology, Faculty of Biology and Environmental Protection, University of Lodz, Lodz, Poland. ${ }^{\circledR}$ email: sylwia. rozalska@biol.uni.lodz.pl 
conditions and that even slight changes in lipidome may affect the functioning of B. bassiana fungi in the natural environment.

\section{Materials and methods}

Reagents. The insecticides used in the study were $\lambda$-cyhalothrin, $\alpha$-cypermethrin, and deltamethrin. Stock solutions of pyrethroids were prepared in $10 \mathrm{mg} \mathrm{mL}^{-1}$ acetonitrile. The other reagents used were phosphatebuffered saline (PBS), propidium iodide, ammonium formate, formic acid, cholesterol, BSTFA/TMCS solution (N,O-bis(trimethylsilyl)trifluoroacetamide/trimethylchlorosilane), DAB (3,3'-diaminobenzidine tetrahydrochloride hydrate), NBT (Nitrotetrazolium Blue chloride), sodium dihydrogen phosphate and disodium hydrogen phosphate dodecahydrate, chloroform, methanol, methyl tert-butyl ether, isopropyl alcohol, methanolic $\mathrm{KOH}, \mathrm{HCl}$, and nitric acid.

Strain and growth conditions. The strain Beauveria bassiana ARSEF 2860 used in the study was obtained from the ARSEF (Agricultural Research Service Collection of Entomopathogenic Fungal Cultures, USA). It was isolated from Schizaphis graminum and can be potentially applied in the biological control of aphids and spider mites ${ }^{13,14}$. Moreover, its genome has been sequenced and is therefore commonly used in scientific research ${ }^{15-17}$. For the experiments, conidia were harvested from 14-day-old cultures of B. bassiana grown on Sabouraud dextrose agar slant and transferred to $40 \mathrm{~mL}$ Sabouraud dextrose broth medium at a density of $10^{6}$ per mL. The medium was supplemented with the abovementioned insecticides at final concentrations of 5, 50, and $100 \mathrm{mg}$ $\mathrm{L}^{-1}$. The inoculated medium was incubated in a rotary shaker $(120 \mathrm{rpm})$ for 24,36 , and $48 \mathrm{~h}$ at $28^{\circ} \mathrm{C}$.

Dry mass measurements, metabolic activity analysis, and microscopy. The effect of pyrethroids on the growth of B. bassiana was determined by measuring the dry mass of cultures. After incubation for 36 and $48 \mathrm{~h}$, the cultures were filtered under reduced pressure through a Whatman membrane filter $(0.45 \mu \mathrm{m})$ and dried at $100^{\circ} \mathrm{C}$ for $1 \mathrm{~h}$ to achieve a constant weight. The effect of pyrethroids on the viability (metabolic activity) of $B$. bassiana was determined by a method using fluorescein diacetate (FDA) with additional modifications ${ }^{18}$. Briefly, the cultures were filtered using a vacuum pump, and the entire wet mass from the flask was divided into portions and transferred to the wells of a 24-well plate. To each well, $1 \mathrm{~mL}$ of FDA in sodium phosphate buffer ( $\mathrm{pH}$ 6.4, $0.7 \mathrm{mg} \mathrm{mL}^{-1}$ ) was added. The samples were incubated in the dark at $28^{\circ} \mathrm{C}$ for $15 \mathrm{~min}$, and the fluorescence of the wells was then read using a plate reader at an excitation wavelength of $485 \mathrm{~nm}$ and an emission wavelength of $530 \mathrm{~nm}$. The fluorescence values were divided by the amount of dry mass estimated for each sample. The results were expressed as \% viability in comparison to the biotic control.

Microscopic observations were made on samples mounted on slides. Images were captured in a Nikon Eclipse E200 microscope equipped with a digital camera and Nikon E Plan objective (40x/0.6) with DeltaPix InSight software.

Determination of sodium, magnesium, potassium, and calcium. The effect of pyrethroids on the content of sodium, magnesium, potassium, and calcium of entomopathogenic fungi was determined in mycelium originating from the insecticide-exposed cultures. Briefly, mycelia were separated from cultures using a filter paper and dried at $100{ }^{\circ} \mathrm{C}$ to obtain dry weight. Subsequently, the biomass was mineralized with concentrated nitric acid (65\%). The content of metals in the samples was estimated by atomic absorption spectrometry (AAS) using a Spectra 240 FS apparatus.

Ergosterol measurement. To determine the content of ergosterol in fungal biomass, $100 \mathrm{mg}$ of biomass was placed in an Eppendorf tube with glass beads. The samples were frozen in liquid nitrogen and then homogenized using a FastPrep-24 instrument for $20 \mathrm{~s}$ at $5 \mathrm{~m} \mathrm{~s}^{-1}$. Ergosterol was extracted as described by Stolarek et al. ${ }^{19}$ with additional modifications. Briefly, $1 \mathrm{~mL}$ of methyl tert-butyl ether-methanol mixture $(3: 1, \mathrm{v} / \mathrm{v})$ was added to the samples and the samples were vortexed. Next, $650 \mu \mathrm{L}$ of water-methanol mixture $(3: 1, \mathrm{v} / \mathrm{v})$ was added, and the samples were vortexed again and centrifuged at $5000 \times g$ for $5 \mathrm{~min}$ at $10^{\circ} \mathrm{C}$. After centrifugation, the top layer was collected and evaporated. Then, the samples were dissolved in a mixture of $0.5 \mathrm{~mL}$ of chloroform, $0.5 \mathrm{~mL}$ of methanolic $\mathrm{KOH}$, and $20 \mu \mathrm{g}$ of cholesterol $\left(20 \mu \mathrm{L}\right.$ from stock solution at the concentration of $\left.1 \mathrm{mg} \mathrm{mL}^{-1}\right)$. After incubating the samples for $1 \mathrm{~h}$ at $23^{\circ} \mathrm{C}, 0.325 \mathrm{~mL}$ of $1 \mathrm{M} \mathrm{HCl}$ and $0.125 \mathrm{~mL}$ of deionized water were added and centrifuged $(5000 \times \mathrm{g})$. Subsequently, the lower layer was transferred to a new Eppendorf tube and dried for $12 \mathrm{~h}$ under fume hood. Then, $100 \mu \mathrm{L}$ of the BSTFA/TMCS solution was added and the samples were incubated for $90 \mathrm{~min}$ at $85^{\circ} \mathrm{C}$. After incubation, $50 \mu \mathrm{L}$ of hexane was added and the samples were transferred to chromatographic tubes. The content of ergosterol in the samples was determined by gas chromatography-tandem mass spectrometry as described previously using an Agilent 7890 system equipped with an HP 5 MS column and a 5975C mass detector ${ }^{20}$.

Phospholipid analysis. For analyzing the phospholipid profile, samples were extracted as described in the previous section. After evaporation, the extract was dissolved in $1 \mathrm{~mL}$ of methanol and analyzed by liquid chromatography-tandem mass spectrometry (LC-MS/MS) using an LC Agilent 1200 system coupled with a Sciex QTRAP 4500 tandem mass spectrometer. A Kinetex C18 column $(50 \mathrm{~mm} \times 2.1 \mathrm{~mm}$, particle size $5 \mu \mathrm{m})$ heated to $40{ }^{\circ} \mathrm{C}$ with a flow rate of $500 \mu \mathrm{L} \mathrm{min}{ }^{-1}$ was used for this purpose. The ion source of the mass spectrometer was operated in a negative mode under the following conditions: spray voltage $4.500 \mathrm{~V}$, curtain gas 25 , nebulizer gas 60 , auxiliary gas 50 , and temperature $600{ }^{\circ} \mathrm{C}$. 
Membrane permeability assay. For determining membrane permeability, $1 \mathrm{~mL}$ of each culture was transferred to an Eppendorf tube and the samples were centrifuged. The supernatant was removed, and $1 \mathrm{~mL}$ of PBS and $2 \mu \mathrm{L}$ of propidium iodide at a concentration of $0.1 \mathrm{mg} \mathrm{mL}^{-1}$ were added. Subsequently, the samples were incubated in the dark at room temperature for $5 \mathrm{~min}$. After incubation, the mycelium was washed twice in PBS, suspended in $1 \mathrm{~mL}$ of PBS, and transferred to a 24-well titration plate. Fluorescence of the samples was measured using a FLUOstar Omega fluorescence microplate reader (excitation wavelength: $540 \mathrm{~nm}$, emission wavelength: $610 \mathrm{~nm}$ ), with the fluorescence of the supernatant set as a background. The results were expressed as a fluorescence unit $(\mathrm{U})$ per mg of dry mass.

Extraction and quantification of pyrethroids. For estimating the content of pyrethroids, the medium was separated from the biomass by filtration and extracted with ethyl acetate followed by methylene chloride. The amount of insecticides in the mycelium and culture medium was determined using a gas chromatographymass spectrometry system equipped with an HP 5 MS column $(30 \mathrm{~m} \times 250 \mu \mathrm{m} \times 0.25 \mu \mathrm{m})$ and a $5975 \mathrm{C}$ mass detector.

Quantification of neutral lipids. Triacylglicerols (TAGs) and diacylglycerols (DAGs) were extracted as described in "Ergosterol measurement" section. After evaporation, the samples were dissolved in $1 \mathrm{~mL}$ of methanol. The content of acylglycerols was determined by LC-MS/MS. To detect acylglycerol, ammonium adducts of multiple reaction monitoring (MRM) scans including parent-daughter pairs were used. Chromatographic separation was conducted on a $\mathrm{C} 18$ column heated to $40^{\circ} \mathrm{C}$, and detection was performed by single-ion monitoring and the enhanced product ion method. Water and a mixture of acetonitrile-isopropyl alcohol (5:2) containing $5 \mathrm{mM}$ ammonium formate and $0.1 \%$ formic acid were used as mobile phases ${ }^{19}$.

Oxidative stress. To determine the content of hydrogen peroxide, $1 \mathrm{~mL}$ of the culture was centrifuged at $2000 \times g$ for $5 \mathrm{~min}$. The obtained biomass was suspended in $1 \mathrm{~mL}$ of DAB solution $\left(1 \mathrm{mg} \mathrm{mL}^{-1}\right.$ of DAB was dissolved in sodium phosphate buffer $(\mathrm{pH} 7.8)$ and $\mathrm{pH}$ was adjusted to 3.8 before adding the solution to the biomass, and the samples were incubated in sunlight for $1 \mathrm{~h}$.

The content of superoxide anion in B. bassiana hyphae was measured using the method described by NykielSzymańska et al. ${ }^{21}$ Briefly, $1 \mathrm{~mL}$ of the culture was centrifuged at $2000 \times g$ for $5 \mathrm{~min}$. The obtained biomass was suspended in $1 \mathrm{~mL}$ of the solution containing $0.1 \% \mathrm{NBT}$ and $10 \mathrm{mM}$ sodium azide dissolved in sodium phosphate buffer ( $\mathrm{pH} 7.8$ ). The samples were incubated for $1 \mathrm{~h}$ in the dark at room temperature.

The content of hydrogen peroxide and superoxide anion in B. bassiana hyphae was examined under an LSM510 Meta confocal laser scanning microscope. The results were expressed as a percentage of the stained area compared to the total hyphal area.

Oosporein determination. To determine the content of oosporein pigment, the cultures were centrifuged at $5000 \times \mathrm{g}$ for $15 \mathrm{~min}$. Then, the medium was acidified to $\mathrm{pH} 2$ with hydrochloric acid. The acidified medium was extracted twice with ethyl acetate for $3 \mathrm{~min}$, and the obtained extracts were dehydrated with anhydrous sodium sulfate. Next, the extracts were filtered through a paper filter, and the filtrate was evaporated using a vacuum evaporator. Subsequently, the samples were dissolved in $1 \mathrm{~mL}$ of methanol and analyzed using an LC Agilent 1200 system coupled with a Sciex QTRAP 3200 tandem mass spectrometer.

Flow injection analysis of samples was conducted with an injection volume of $10 \mu \mathrm{L}$ and a flow rate of $600 \mu \mathrm{L}$ $\mathrm{min}^{-1}$. A mixture of water with $0.1 \%$ formic acid (solvent A) and a mixture of acetonitrile with $0.1 \%$ formic acid (solvent B) were used as mobile phases. A phase A-phase B ratio of 30:70 was maintained for 0.7 min. Tandem mass spectrometry detection was conducted using the electrospray ion source ESI with negative polarization. To confirm the presence of oosporein, the three following MRM pairs were determined: Q1/Q3-305.1/177.1, $305.1 / 149.1$, and 305.1/133.1. Of these, the quantitative pair was 305.1/177.1 The individual analytes parameters were: entrance potential (EP; (-5)), collision cell entrance potential (CEP; (-30)), collision energy (CE; (-30)), collision cell exit potential (CXP; (-1)), declustering potential (DP (-35)). The amount of oosporein was determined based on a calibration curve over a concentration range of $1-100 \mu \mathrm{g}$, with quadratic regression, where the " $R$ " value was 0.9954 .

Statistical analyses. All samples were prepared in triplicate. The experiments were repeated twice, and the standard deviation (SD) of the results was determined. The normality of the data distribution was determined by the Shapiro-Wilk test. Data were analyzed using one-way analysis of variance (ANOVA), and the means were compared using Tukey's post hoc test. A $p$-value of $<0.05$ was considered statistically significant. Statistical analyses were conducted in the STATISTICA version 13.3 software (StatSoft).

\section{Results and discussion}

Effect of pyrethroids on B. bassiana dry biomass content and metabolic activity. After $36 \mathrm{~h}$ of incubation, statistically significant $(P<0.01)$ changes in biomass dry weight were observed in the B. bassiana samples supplemented with pyrethroids at the concentrations of 50 and $100 \mathrm{mg} \mathrm{L}^{-1}$ (Supplementary Table S1). However, after $48 \mathrm{~h}$ of incubation, statistically significant changes were noted only in the samples supplemented with $100 \mathrm{mg} \mathrm{L}^{-1}$ of $\lambda$-cyhalothrin $(P=0.00169)$ and $\alpha$-cypermethrin $(P=0.015635)$. The results of our study also indicated that the tested pyrethroids did not affect the growth of $B$. bassiana at the concentration of $5 \mathrm{mg} \mathrm{L}^{-1}$.

Intriguingly, microscopic observations did not reveal any morphological changes in mycelia exposed to pyrethroids (Supplementary Fig S1 and Supplementary Fig S2) but we noted a significant decrease in the number 


\begin{tabular}{|l|l|l|l|c|}
\hline & \multirow{4}{*}{} & \multicolumn{3}{|l|}{ Pyrethroids $\left(\mathbf{1 0 0} \mathbf{~ m g ~ L}^{-1}\right)$} \\
\cline { 3 - 5 } & Control & $\lambda$-cyhalothrin & $\alpha$-cypermethrin & Deltamethrin \\
\hline $24 \mathrm{~h}$ & $100.00 \pm 4.76$ & $135.73 \pm 6.58^{* *}$ & $132.46 \pm 19.00^{* *}$ & $146.85 \pm 12.33^{* *}$ \\
\hline $36 \mathrm{~h}$ & $100.00 \pm 3.85$ & $111.11 \pm 11.76^{*}$ & $88.76 \pm 12.80$ & $98.26 \pm 20.45$ \\
\hline $48 \mathrm{~h}$ & $100.00 \pm 12.39$ & $113.59 \pm 27.11$ & $119.37 \pm 17.51$ & $120.79 \pm 15.00$ \\
\hline
\end{tabular}

Table 1. Metabolic activity (\%) of B. bassiana after 24,36 , and $48 \mathrm{~h}$ of culture with $\lambda$-cyhalothrin, $\alpha$-cypermethrin, and deltamethrin. Data are means \pm SD; all samples were prepared in triplicate, and the experiments were repeated twice. Results were tested by one-way ANOVA; significance: ${ }^{* *} P<0.01,{ }^{\star} P<0.05$.

\begin{tabular}{|c|c|c|c|c|c|c|c|c|c|c|}
\hline \multirow{3}{*}{$\begin{array}{l}\text { Element } \mathrm{mg} \mathrm{g}^{-1} \text { dry } \\
\text { mass }\end{array}$} & \multirow[b]{3}{*}{ Control } & \multicolumn{9}{|c|}{ Pyrethroids $\left(\mathrm{mg} \mathrm{L}^{-1}\right)$} \\
\hline & & \multicolumn{3}{|l|}{$\lambda$-cyhalothrin } & \multicolumn{3}{|c|}{ a-cypermethrin } & \multicolumn{3}{|l|}{ Deltamethrin } \\
\hline & & 5 & 50 & 100 & 5 & 50 & 100 & 5 & 50 & 100 \\
\hline $\mathrm{Na}$ & $2.68 \pm 0.11$ & $2.54 \pm 0.18$ & $1.84 \pm 0.18^{\star *}$ & $1.31 \pm 0.10^{* *}$ & $1.94 \pm 0.24^{\star *}$ & $1.64 \pm 0.02^{\star *}$ & $1.73 \pm 0.03^{\star *}$ & $1.50 \pm 0.18^{\star *}$ & $1.39 \pm 0.27^{\star *}$ & $1.26 \pm 0.22^{* *}$ \\
\hline $\mathrm{Mg}$ & $0.28 \pm 0.02$ & $0.19 \pm 0.04^{* *}$ & $0.27 \pm 0.03$ & $0.34 \pm 0.03^{*}$ & $0.18 \pm 0.01^{\star *}$ & $0.22 \pm 0.00^{*}$ & $0.22 \pm 0.01^{*}$ & $0.31 \pm 0.00$ & $0.21 \pm 0.00^{* *}$ & $0.26 \pm 0.02$ \\
\hline $\mathrm{K}$ & $11.28 \pm 0.03$ & $11.71 \pm 0.52$ & $8.77 \pm 0.53^{\star *}$ & $7.43 \pm 0.25^{\star *}$ & $8.47 \pm 0.37^{\star *}$ & $8.64 \pm 0.55^{\star *}$ & $10.64 \pm 0.91$ & $11.32 \pm 0.24$ & $9.13 \pm 0.06^{* *}$ & $8.79 \pm 0.61^{\star *}$ \\
\hline $\mathrm{Ca}$ & $2.46 \pm 0.06$ & $2.45 \pm 0.09$ & $2.06 \pm 0.10^{* *}$ & $2.25 \pm 0.14$ & $2.15 \pm 0.01^{\star *}$ & $2.12 \pm 0.14^{\star *}$ & $2.26 \pm 0.09$ & $2.16 \pm 0.04^{\star *}$ & $1.90 \pm 0.11^{\star *}$ & $1.68 \pm 0.15^{\star *}$ \\
\hline
\end{tabular}

Table 2. Content of sodium, magnesium, potassium, and calcium in the B. bassiana cells after cultivation with $\lambda$-cyhalothrin, $\alpha$-cypermethrin, and deltamethrin. Data are means \pm SD; all samples were prepared in triplicate, and the experiments were repeated twice. Results were tested by one-way ANOVA; significance: ${ }^{* *} P<0.01$, ${ }^{\star} P<0.05$.

of blastospores incubated for 24 and $48 \mathrm{~h}$ with pesticides at the concentrations of 50 and $100 \mathrm{mg} \mathrm{L}^{-1}$ (Supplementary Table S2). Among the tested insecticides, cyhalothrin had the most detrimental effect on this process. According to our knowledge, there are no data regarding the effect of pyrethroids on blastospores formation in liquid cultures. However, in the cultures on solid media, a decrease in the sporulation of $B$. bassiana was noted in the presence of cypermethrin and deltamethrin ${ }^{22}$. Inhibition of B. bassiana sporulation by herbicides in solid media has also been documented ${ }^{23}$. It seems that xenobiotics quite often inhibit the conidiation process, but the molecular mechanisms of this phenomenon are still unknown.

The study analyzed a wide range of insecticides concentrations, which were selected based on manufacturers' recommended doses for field applications. The lowest concentration tested in the study $\left(5 \mathrm{mg} \mathrm{L}^{-1}\right)$ is tenfold lower than that recommended for commercial preparations for application per $100 \mathrm{~m}^{2}$ of the area. The concentration $50 \mathrm{mg} \mathrm{L}^{-1}$ is within the recommended concentration range of cypermethrin and deltamethrin but exceeds that of cyhalothrin. The highest tested concentration $\left(100 \mathrm{mg} \mathrm{L}^{-1}\right)$ is twice as high as the recommended concentration of cyhalothrin and deltamethrin. Because cypermethrin is used at high doses in the field, this concentration does not exceed the range of commonly applied range of doses.

The data reported in the literature are in line with our results. Pyrethroids used in recommended (and higher) doses strongly inhibited entomopathogenic fungi during the initial period of growth, limiting the rate of colony formation by more than $40 \%$, while at lower doses these insecticides had a minimal inhibitory effect ${ }^{24}$.

We also checked whether the highest tested insecticide concentration influenced the metabolic activity of entomopathogenic fungi as previous studies have only analyzed the effect of pyrethroids on their growth. The tests conducted in our study did not show any negative effect of pyrethroids on the metabolic activity of $B$. bassiana, and even a slight increase in activity was found in the samples exposed to the tested substances (Table 1). In 24-h cultures, a 1.47-fold increase in metabolic activity was noted in the presence of deltamethrin $(P=0.000176)$, 1.36 -fold increase in the presence of $\lambda$-cyhalothrin $(P=0.000237)$, and 1.32 -fold increase in the presence of a-cypermethrin $(P=0.000376)$. In 36-h cultures, a 1.1-fold increase in metabolic activity was observed in the presence of $\lambda$-cyhalothrin $(P=0.016223)$; however, in the presence of $\alpha$-cypermethrin and deltamethrin, the metabolic activity in the samples was comparable to that observed in the control. In 48-h cultures, no differences in metabolic activity were found between the cultures exposed to pyrethroids and the biotic control.

Despite the growth inhibition, the tested insecticides did not adversely affect the metabolic activity of $B$. bassiana. This finding is different from the previous observations because most of the toxic compounds studied so far have been observed to cause a simultaneous reduction in the growth and viability of microorganisms. Różalska et al. ${ }^{18}$ used the same method in their study to examine the metabolic activity of Metarhizium robertsii, another entomopathogenic fungal species. The authors found that the addition of both 4-n-nonylphenol and technical nonylphenol caused a statistically significant decrease in the metabolic activity of the tested strain.

Effect of pyrethroids on the content of sodium, potassium, calcium, and magnesium in biomass. All tested insecticides caused a decrease in sodium content in the biomass of B. bassiana (Table 2). The addition of $100 \mathrm{mg} \mathrm{L}^{-1}$ of $\lambda$-cyhalothrin and 50 and $100 \mathrm{mg} \mathrm{L}^{-1}$ of deltamethrin resulted in a two-fold reduction in the amount of this element in the fungal cells. To our knowledge, our study is the first to describe this 


\section{Content of ergosterol in cultures of $\boldsymbol{B}$. bassiana}

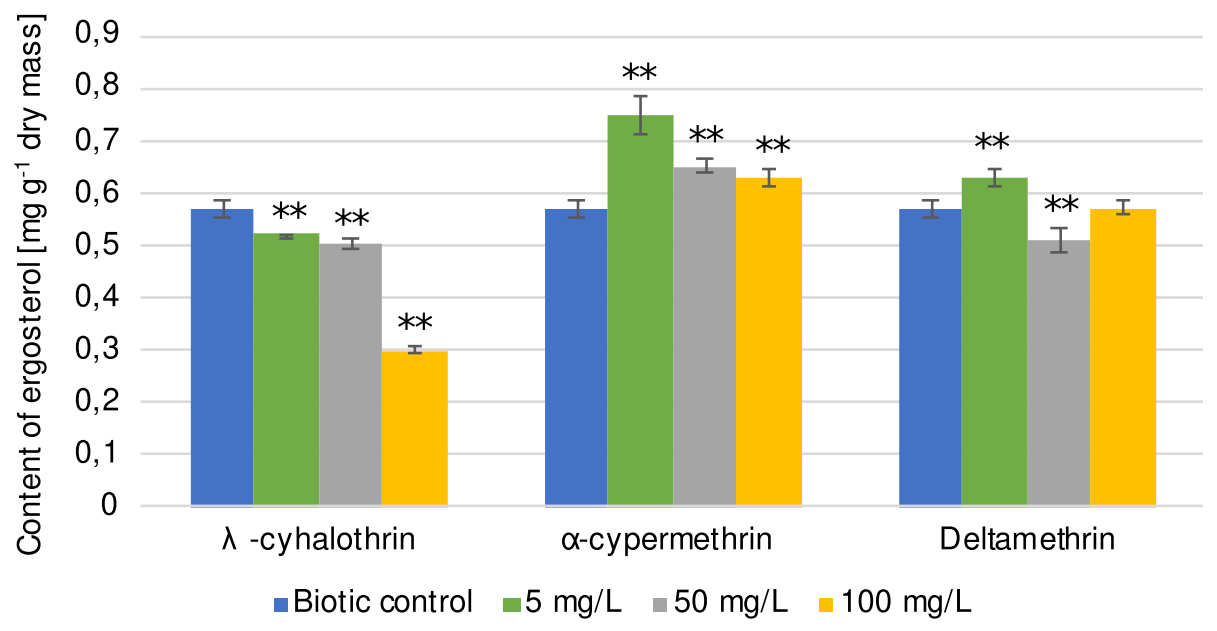

Figure 1. Content of ergosterol [ $\mathrm{mg} \mathrm{g}^{-1}$ dry mass] in the B. bassiana cells after $48 \mathrm{~h}$ of cultivation with $\lambda$-cyhalothrin, $\alpha$-cypermethrin, and deltamethrin. All samples were prepared in triplicate, and the experiments were repeated twice. Results were tested by one-way ANOVA; significance: ${ }^{\star \star} P<0.01,{ }^{\star} P<0.05$.

phenomenon for fungi. The insecticidal action of pyrethroids is based on their binding to sodium voltage-gated channels in the neuronal membranes of insects ${ }^{25}$. Interestingly, it has been postulated that genes coding sodium voltage-gated channels are not present in the fungal genomes ${ }^{26}$ and the role of other sodium-transporting channels in fungi is still being elucidated.

The presence of pyrethroids was also found to influence the content of other elements in B. bassiana biomass (Table 2). Cypermethrin (even at low concentration) decreased the levels of $\mathrm{K}, \mathrm{Mg}$, and $\mathrm{Ca}$, while deltamethrin caused a decrease in their levels in fungal cells at a concentration of $50 \mathrm{mg} \mathrm{L}^{-1}$. Changes in the endogenous level of these elements might have resulted from increased membrane permeability after the exposure of fungal cells to pyrethroids.

A similar effect or leakage of potassium ions from the cells of Candida albicans associated with an increase in membrane permeability was observed by Lee and Lee $(2014)^{27}$ after the treatment of cells with curcumin. The results of their study indicated that pyrethroids cause disturbances in the ionic balance not only in insects, but also in entomopathogenic fungi. On the other hand, our results showed that pyrethroids did not affect the viability of $B$. basiana. Therefore, further research is needed to elucidate the mechanism that enables the fungi to remain viable in the presence of pyrethroids, despite the negative impact of these compounds on the integrity of the cell membrane.

Effect of pyrethroids on the ergosterol content in entomopathogenic fungi. The changes in the elemental content in fungal biomass may be associated with changes in the permeability of cell membranes. Ergosterol is an important marker of cell response to toxic substances. It plays a key role in many cell processes and is crucial for the integrity and functioning of the plasma membrane $e^{20}$.

Our study showed that the studied pyrethroids differed in their effects on the ergosterol content of $B$. bassiana cells. $\lambda$-cyhalothrin, at all tested concentrations $\left(5,50\right.$, and $\left.100 \mathrm{mg} \mathrm{L}^{-1}\right)$, caused a statistically significant decrease in content (by $8.78 \%(P=0.000190), 12.29 \%(P=0.000163)$, and $47.37 \%(P=0.000163)$, respectively), in comparison to the biotic control), while $\alpha$-cypermethrin caused an increase in its synthesis in $B$. bassiana cells (Fig. 1). In the case of deltamethrin, an increase in the ergosterol amount was observed at the concentration of $5 \mathrm{mg} \mathrm{L}^{-1}$, but at $50 \mathrm{mg} \mathrm{L}^{-1}$ a slight reduction in this component was observed in the fungal cells.

The literature has no information regarding the modifications in ergosterol level in the presence of pyrethroids in organisms, but the results observed in the content of elements and ergosterol in this work suggest that pyrethroids affect the cell membranes of B. bassiana.

Among the examined pyrethroids, the strongest membrane permeability in B. bassiana cells was found in presence of $\lambda$-cyhalothrin. Moreover, the lowest levels of ergosterol and higher phosphatidylcholine (PC)/ phosphatidylethanolamine (PE) ratio were noticed in the cells in the presence of this insecticide. Considering the fact that ergosterol, PC, and PE regulate membrane permeability and fluidity, it seems that a decrease in the levels of ergosterol and PE caused an increase in the membrane permeability of fungal cells.

Effect of pyrethroids on phospholipid compositions. The most important phospholipid classes determined in the B. bassiana membranes are presented in Table 3. Quantitative lipidomic analyses revealed the highest content of PC (51.08\%) and PE (45.48\%) in the membranes. The contents of phosphatidylinositol (PI), phosphatidylserine (PS), and phosphatidic acid (PA) was estimated as $2.78 \%, 0.58 \%$, and $0.08 \%$, respectively. In a previous study that examined the phospholipid profile of $B$. bassiana, PC was identified as the dominant class of phospholipids, followed by $\mathrm{PE}$, the amount of which was found to be half of that of $\mathrm{PC}^{28}$. Other phospholipid 


\begin{tabular}{|c|c|c|c|c|}
\hline \multirow[b]{2}{*}{ Phospholipid class } & \multirow[b]{2}{*}{ Control } & \multicolumn{3}{|c|}{ Pyrethroids (100 mg L $\left.{ }^{-1}\right)$} \\
\hline & & $\lambda$-cyhalothrin & a-cypermethrin & Deltamethrin \\
\hline PA relative abundance (\%) & $0.08 \pm 0.03$ & $0.06 \pm 0.01$ & $0.09 \pm 0.03$ & $0.07 \pm 0.01$ \\
\hline PC relative abundance (\%) & $51.08 \pm 1.35$ & $54.66 \pm 0.37^{* *}$ & $53.38 \pm 0.92^{* *}$ & $53.76 \pm 0.57^{* *}$ \\
\hline PE relative abundance (\%) & $45.48 \pm 1.34$ & $40.83 \pm 0.74^{* \star}$ & $42.18 \pm 0.94^{\star \star}$ & $42.20 \pm 0.51^{* *}$ \\
\hline PI relative abundance (\%) & $2.78 \pm 0.07$ & $3.85 \pm 0.48^{* *}$ & $3.68 \pm 0.32^{\star *}$ & $3.49 \pm 0.19^{* *}$ \\
\hline PS relative abundance (\%) & $0.58 \pm 0.08$ & $0.60 \pm 0.10$ & $0.66 \pm 0.05$ & $0.49 \pm 0.09$ \\
\hline $\mathrm{PC} / \mathrm{PE}$ & $1.12 \pm 0.06$ & $1.34 \pm 0.03^{* *}$ & $1.27 \pm 0.05^{\star *}$ & $1.27 \pm 0.03^{* *}$ \\
\hline $\mathrm{PI} / \mathrm{PS}$ & $4.84 \pm 0.83$ & $6.58 \pm 1.90$ & $5.59 \pm 0.89$ & $7.37 \pm 1.95^{\star}$ \\
\hline
\end{tabular}

Table 3. Phospholipid composition and PC/PE and PI/PS ratios determined in the B. bassiana cells after $48 \mathrm{~h}$ of cultivation with $\lambda$-cyhalothrin, $\alpha$-cypermethrin, and deltamethrin. Data are means $\pm S D$; all samples were prepared in triplicate, and the experiments were repeated twice. Results were tested by one-way ANOVA; significance: ${ }^{\star *} P<0.01,{ }^{\star} P<0.05$.

classes (PS, PI, and PA) were also detected, with the content of PA being the lowest, as was also observed in our work.

The lipidomic data of this study revealed that all tested pyrethroids affected the phospholipid profile of $B$. bassiana and caused a statistically significant increase in the PC content with a simultaneous decrease in the PE content (Table 3). As PC and PE are the main phospholipids in B. bassiana cells, the membrane dynamics of the cells depends on their ratio. PC is responsible for stabilizing the membrane bilayer phase, and maintaining its structure and functionality, while PE forms nonbilayer hexagonal phases. Changes in the phospholipid profile thus affect the properties of cell membranes ${ }^{19}$. The PC/PE ratio is also an essential factor of membrane integrity ${ }^{29}$. In this study, all the tested insecticides significantly increased this ratio $(P=0.000175, P=0.000201, P=0.000186$ in the presence of $\lambda$-cyhalothrin, $\alpha$-cypermethrin, and deltamethrin, respectively), thereby leading to higher membrane fluidity. In addition, the increased PC/PE ratios were in concordance with the changes observed in the endogenous levels of $\mathrm{K}, \mathrm{Mg}$, and $\mathrm{Ca}$.

The LC-MS/MS analysis revealed that PC 18:2 18:2, PC 18:2 18:1, and PE 16:0 18:2 were the major lipids in B. bassiana cells and that pyrethroids had a noticeable influence on their content (Supplementary Table S3). A statistically significant increase in the level of unsaturated PC 18:2 18:2 was noted in the presence of $\lambda$-cyhalothrin (34.49 $\pm 0.64 \%, P=0.000232)$, $\alpha$-cypermethrin $(33.85 \pm 0.43 \%, P=0.000238)$, and deltamethrin $(33.15 \pm 0.39 \%$, $P=0.000289)$, compared to the biotic control (29.88 $\pm 0.25 \%)$. A higher amount of unsaturated PC shown by the analyses may also suggest an increase in membrane permeability. The level of PE 16:0 18:2, which was identified as the most predominant phospholipid, decreased to $18.04 \pm 1.54 \%$ in the presence of $\lambda$-cyhalothrin $(P=0.005753)$, $18.62 \pm 2.44 \%$ in the presence of $\alpha$-cypermethrin $(P=0.024558)$, and $18.89 \pm 1.30 \%$ in the presence of deltamethrin $(P=0.047449)$, compared to the biotic control $(21.34 \pm 1.11 \%)$.

All tested pyrethroids also caused a statistically significant increase $(P<0.001)$ in the PI content. The amount of PI 16:0 18:2 increased after the addition of $\lambda$-cyhalothrin $(P=0.005384)$, and that of PI 18:2 18:2 increased in the presence of all tested pyrethroids $(P=0.000178, P=0.000181, P=0.000302$ with $\lambda$-cyhalothrin, $\alpha$-cypermethrin, and deltamethrin, respectively) (Supplementary Table S3). PI acts as a precursor for the synthesis of many important lipid compounds, such as complex sphingolipids, phosphoinositides and inositol polyphosphates, and thus plays a key role in the basic metabolism. It is also essential for the anchoring of glycolipids in the plasma membrane ${ }^{30}$. Previous works have also demonstrated that toxic substances such as tributyltin caused an increase in PI content in fungal cell membranes ${ }^{30}$. No significant changes were observed in the content of PS (a precursor of PC and PE) and PA (a signal lipid) in this work.

Effect of pyrethroids on acylglycerols content in entomopathogenic fungi. Neutral lipids, TAGs and DAGs, are the main reserve material and important element of the phospholipids biosynthesis pathway ${ }^{31}$. The present study revealed that pyrethroids reduced the total amount of acylglycerols and the content of DAGs was higher than that of TAGs in B. bassiana cells (Fig. 2). The most significant reduction in TAGs was observed in the samples exposed to $\alpha$-cypermethrin, while $\lambda$-cyhalothrin caused the greatest decrease in the content of DAGs. It has been found that the precursor of acylglycerols in the TAG biosynthesis pathway is glycerol-3-phosphosphate, which is converted to lysophosphatidic acid and subsequently to phosphatidic acid. In the next steps, phosphatidic acid is converted to DAG, and then DAG is converted to TAG ${ }^{32}$. No data can be found in the literature regarding the inhibition of acylglycerol synthesis by pyrethroids in entomopathogenic fungi. Moreover, in Donax trunculus clam, a reverse effect has been observed after the exposure of the organisms to pyrethroids ${ }^{33}$.

Using LC-MS/MS, 13 TAG and 9 DAG species were identified in the B. bassiana samples (Supplementary Table S4). The major molecular TAGs detected were: 52:2, 54:3, 54:4, and 54:6 and DAGs were 34:2, $36: 1$, and $36: 2$. In the presence of pyrethroids, some changes in the content of individual neutral lipids were recorded. $\lambda$-Cyhalothrin caused a statistically significant decrease in the content of TAGs 52:4 $(P=0.041826)$, 54:1 $(P=0.000199), 54: 2(P=0.000199), 54: 3(P=0.002386), 54: 4(P=0.000203)$, and $54: 6(P=0.000338)$ and an increase in TAGs 48:0 $(P=0.000199), 50: 0(P=0.000199), 52: 1(P=0.000199)$, and 52:2 $(P=0.000239)$. a-Cypermethrin caused a statistically significant decrease in the content of TAGs 52:3 $(P=0.000417), 52: 4$ $(P=0.018714), 54: 1 \quad(P=0.000199), 54: 2(P=0.000199), 54: 3(P=0.000250), 54: 4(P=0.002145)$, and $54: 6$ $(P=0.000241)$ with a simultaneous increase in the content of TAGs 48:0 $(P=0.000199), 50: 0(P=0.000199)$, 
Content of acylglycerols in cultures of $B$. bassiana

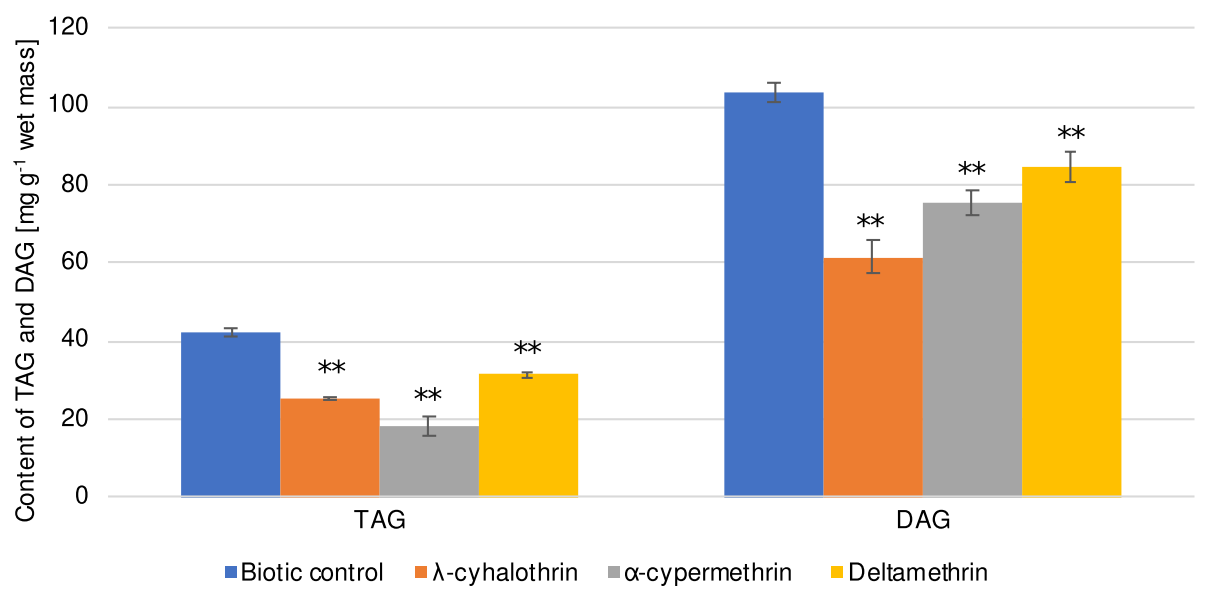

Figure 2. Content of tri- and diacylglycerols in the B. bassiana cells after $48 \mathrm{~h}$ of cultivation with $\lambda$-cyhalothrin, a-cypermethrin and deltamethrin at a concentration of $100 \mathrm{mg} \mathrm{L}^{-1}$. All samples were prepared in triplicate, and the experiments were repeated twice. Results were tested by one-way ANOVA; significance: ${ }^{\star *} P<0.01,{ }^{\star} P<0.05$.

\begin{tabular}{|l|l|c|l|l|}
\hline & \multirow{4}{*}{ Control } & \multicolumn{4}{|l|}{ Pyrethroids $\left(\mathbf{1 0 0} \mathbf{~ m ~ L ~}^{-1}\right)$} \\
\cline { 3 - 5 } & $\lambda$-cyhalothrin & $\boldsymbol{\alpha}$-cypermethrin & Deltamethrin \\
\hline Membrane permeability [\%] & $100.00 \pm 5.77$ & $123.51 \pm 4.49^{* *}$ & $115.69 \pm 4.10^{*}$ & $107.00 \pm 1.80$ \\
\hline Recovery [\%] & & & & \\
\hline Culture medium & - & $1.93 \pm 0.24$ & $2.50 \pm 0.12$ & $1.66 \pm 0.33$ \\
\hline Mycelium & - & $71.84 \pm 1.12$ & $70.16 \pm 0.83$ & $99.64 \pm 0.74$ \\
\hline
\end{tabular}

Table 4. Membrane permeability and accumulation of pyrethroids in the B. bassiana cells. Data are means $\pm \mathrm{SD}$; all samples were prepared in triplicate, and the experiments were repeated twice. Results were tested by one-way ANOVA; significance: ${ }^{* *} P<0.01,{ }^{\star} P<0.05$.

and 52:1 $(P=0.000199)$. Our results indicate that deltamethrin had the weakest effect on individual TAG species and some changes were only recorded in the content of TAGs 54:0 $(P=0.000270)$ and 54:2 $(P=0.000199)$ and DAG 36:1 $(P=0.033229)$.

$\lambda$-Cyhalothrin and $\alpha$-cypermethrin increased the amounts of DAGs $32: 0(P=0.000199 ; P=0.009261)$, and 34:2 ( $P=0.000278 ; P=0.000199)$; however, a higher content of DAG 34:0 $(P=0.005059)$ was also noticed in the $B$. bassiana samples cultivated with $\lambda$-cyhalothrin. Interestingly, the abundance of DAG 36:1 was found to be clearly reduced in the presence of $\lambda$-cyhalothrin $(P=0.000210)$ and $\alpha$-cypermethrin $(P=0.000254)$, while deltamethrin caused an increase in the content of this acylglycerol $(P=0.033229)$.

Our results suggest that $\lambda$-cyhalothrin and $\alpha$-cypermethrin increased the content of saturated TAGs and DAGs and decreased the content of unsaturated TAGs. Deltamethrin had a slight effect on the amounts of individual TAGs and DAGs.

Compared to the control, the lowest TAG/DAG ratio was found in the presence of cypermethrin $(P=0.003244)$, which may indicate increased accumulation of DAG related to TAG inhibition (Supplementary Table S4).

TAGs are the basic reserve material for cells and together with DAG they constitute an important element of phospholipid biosynthesis ${ }^{19,34}$ and also play a crucial role in protecting cells against oxidative damage ${ }^{35}$. Studies in mammals showed that accumulation of TAGs in lipid droplets prevents oxidative stress in cells by limiting the generation of reactive oxygen species (ROS) ${ }^{35}$. On the other hand, in this study, the fungal cells exposed to pyrethroids had a lower accumulation of TAGs accompanied by an increased percentage of hyphae containing superoxide anion radicals and hydrogen peroxide.

TAGs are also involved in fungal virulence, supporting the penetration of fungi into the insect cuticle ${ }^{29,34}$. This suggests that pyrethroids affect the metabolism of fungi, which play an important ecological role in the environment, controlling the insect populations.

Effect of pyrethroids on membrane permeability and their accumulation. The membrane permeability of $B$. bassiana cells exposed to pyrethroids was determined by intracellular accumulation of propidium iodide (Table 4). The results showed a statistically significant increase in red fluorescence in fungal biomass incubated in the presence of $\lambda$-cyhalothrin and $\alpha$-cypermethrin at the concentration of $100 \mathrm{mg} \mathrm{L}^{-1}$ 


\begin{tabular}{|c|c|c|c|c|c|}
\hline & & \multirow[b]{2}{*}{ Control } & \multicolumn{3}{|c|}{ Pyrethroids $\left(100 \mathrm{mg} \mathrm{L}^{-1}\right)$} \\
\hline & & & $\lambda$-cyhalothrin & a-cypermethrin & Deltamethrin \\
\hline \multirow{3}{*}{$\mathrm{O}_{2}^{--}$} & $24 \mathrm{~h}$ & $1.76 \pm 1.26$ & $1.93 \pm 1.02$ & $4.09 \pm 2.42^{\star}$ & $2.37 \pm 0.74$ \\
\hline & $36 \mathrm{~h}$ & $0.55 \pm 0.08$ & $4.73 \pm 0.82^{* *}$ & $10.97 \pm 4.15^{\star *}$ & $7.34 \pm 1.44^{* *}$ \\
\hline & $48 \mathrm{~h}$ & $1.51 \pm 1.02$ & $7.87 \pm 3.25^{*}$ & $18.62 \pm 3.22^{* *}$ & $22.80 \pm 5.31^{\star *}$ \\
\hline \multirow{3}{*}{$\mathrm{H}_{2} \mathrm{O}_{2}$} & $24 \mathrm{~h}$ & nd & nd & nd & nd \\
\hline & $36 \mathrm{~h}$ & $0.14 \pm 0.00$ & $0.22 \pm 0.06$ & $0.63 \pm 0.31^{\star *}$ & $0.37 \pm 0.12$ \\
\hline & $48 \mathrm{~h}$ & $0.33 \pm 0.26$ & $3.80 \pm 0.59^{* *}$ & $4.64 \pm 2.00^{* *}$ & $3.28 \pm 0.99^{* *}$ \\
\hline
\end{tabular}

Table 5. Percentage of hyphae containing superoxide anion radicals and hydrogen peroxide in B. bassiana after incubation with $\lambda$-cyhalothrin, $\alpha$-cypermethrin, and deltamethrin. Data are means $\pm S D$; all samples were prepared in triplicate, and the experiments were repeated twice. Results were tested by one-way ANOVA; significance: ${ }^{*} P<0.01,{ }^{\star} P<0.05$. nd - not detected.

(by $23.5 \%, P=0.000196$ and $15.7 \%, P=0.000328$, respectively) and a slight increase in the cultures containing deltamethrin at the concentration of $100 \mathrm{mg} \mathrm{L}^{-1}(7 \%)$. A statistically significant increase in the PC/PE ratio was also confirmed (Table 3). Furthermore, a higher content of ergosterol was noted in the samples supplemented with a-cypermethrin and deltamethrin, which proved an increase in the fluidity of B. bassiana cell membranes (Fig. 1).

Previous studies have also reported the changes in the fungal membranes permeability caused by pesticides. Higher membrane permeability associated with an increase in the PC/PE ratio was described for several strains of Trichoderma spp. cultured with chloroacetanilide herbicides ${ }^{21}$. Similarly, increased membrane fluidity was observed for the herbicide degrader Umbelopsis isabellina in the presence of 2,4- $\mathrm{D}^{36}$. Increased membrane fluidity was found to facilitate the transport of toxic substances into the fungal cells, which causes the accumulation or even degradation of these substances ${ }^{7}$.

In this study, all tested pyrethroids were found to be accumulated in the mycelium of B. bassiana. The amounts of $\lambda$-cyhalothrin and $\alpha$-cypermethrin in fungal biomass were estimated at $71.84 \%$ and $70.16 \%$, respectively, while deltamethrin was accumulated at $99.64 \%$. This suggests that increased membrane permeability promotes the penetration of pyrethroids into the mycellium.

Effect of pyrethroids on ROS production. Literature data indicate that the mechanism of toxicity of insecticides could be associated with enhanced intracellular ROS production/antioxidant enzyme activity ${ }^{8}$. Therefore, in this study, we determined the intracellular level of superoxide anion radicals and hydrogen peroxide in B. bassiana mycelia following exposure to pyrethroids (Table 5).

At the beginning of the experiment (24-h incubation), a slight increase in the level of superoxide anion radicals was observed only in the samples cultivated with a -cypermethrin, while $\mathrm{H}_{2} \mathrm{O}_{2}$ was not detected in the biomass. It has been found that under stress conditions, superoxide anion radicals appear first and are then converted by superoxide dismutase to hydrogen peroxide. In the present study, prolonging the incubation time resulted in a significant increase in the ROS levels. In the 36-h biomass, the amount of superoxide anion radicals increased by eightfold, almost 20 -fold and 13 -fold in the presence of $\lambda$-cyhalothrin $(P=0.008298)$, a-cypermethrin $(P=0.000161)$, and deltamethrin $(P=0.000194)$, respectively. Similarly, in 48 -h cultures, approximately fivefold $(P=0.011729), 12$-fold $(P=0.000175)$, and 15-fold $(P=0.000175)$ increase in the level of $\mathrm{O}_{2}{ }^{--}$were noted. A statistically significant increase in hydrogen peroxide by 11 -fold, 14-fold, and ninefold was detected at $48 \mathrm{~h}$ of incubation in the samples exposed to $\lambda$-cyhalothrin $(P=0.000231)$, $\alpha$-cypermethrin $(P=0.000177)$, and deltamethrin $(P=0.000585)$, respectively. These results clearly highlight that oxidative stress was induced in $B$. bassiana after the exposure to pyrethroids. Oxidative stress may have been caused by the entry and accumulation of pyrethroids in mycelium, which, as mentioned earlier, may be correlated with the increase in membrane permeability. Furthermore, the observed decrease of TAGs in the presence of pyrethroids may influence the generation of free radicals, the level of which may also depend on the amount of TAGs in cells.

Due to their lifestyle, entomopathogenic fungi may often experience oxidative stress by different factors, including exposure to insect host defense such as production of $\operatorname{ROS}^{37}$. Therefore, they are characterized by extremely flexible metabolism which enables them to grow under various environmental conditions. The results of our work confirmed that pyrethroids might be one of the harmful factors, which is in line with the observation made so far in fish and rats that oxidative stress is induced by these substances ${ }^{38,39}$.

Effect of pyrethroids on secondary metabolite production by Beauveria species. Oosporein, a secondary metabolite belonging to the octadepsipeptide group, exhibits insecticidal, antiviral, and antibacterial effects $^{40}$. It is an important metabolite of entomopathogenic fungi and stimulates infection in insects ${ }^{7}$. In our study, a lack of oosporein production was observed in the 24-h culture and the presence of pyrethroids was found to inhibit the pigment production even in the later period of incubation. In 36-h cultures, the content of oosporein was decreased in the samples exposed to pyrethroids than in the biotic control $(\lambda$-cyhalothrin: 6.7 fold $(P=0.000254)$, $\alpha$-cypermethrin: 11.3 -fold $(P=0.000242)$, deltamethrin: 7.5 -fold $(P=0.000250))$ (Fig. 3). In the 48 -h culture, a slight increase in pigment content was observed in the samples with all tested insecticides, but these differences were not statistically significant (Fig. 3). 


\section{Content of oosporein in cultures of $B$. bassiana}

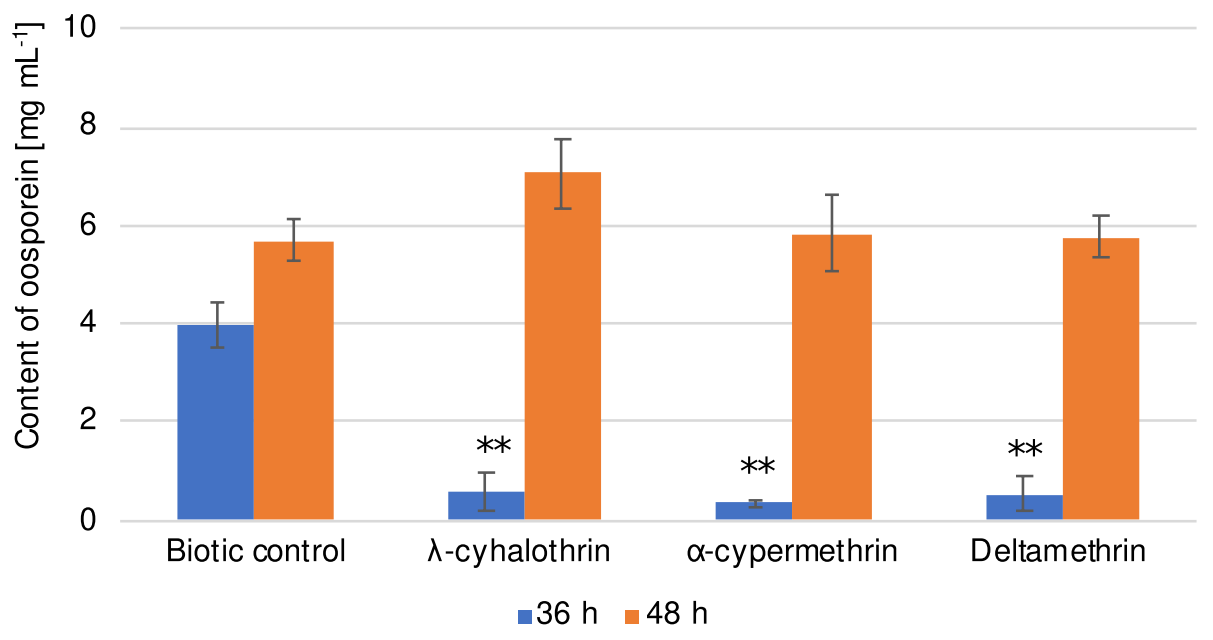

Figure 3. Content of oosporein $\left[\mathrm{mg} \mathrm{mL}^{-1}\right]$ in B. bassiana after 36 and $48 \mathrm{~h}$ of cultivation with $\lambda$-cyhalothrin, a-cypermethrin, and deltamethrin at a concentration of $100 \mathrm{mg} \mathrm{L}^{-1}$. All samples were prepared in triplicate, and the experiments were repeated twice. Results were tested by one-way ANOVA; significance: ${ }^{\star \star} P<0.01,{ }^{\star} P<0.05$.

In general, microorganisms produce secondary metabolites to protect them against both biotic and abiotic stress $^{41}$. The influence of toxic compounds on the production of these metabolites has been poorly understood, and so far only the inhibition of destruxins production by Metarhizium sp. by acetamiprid has been proved ${ }^{42}$. Temporary inhibition of oosporein production in the presence of pyrethroids indicates a harmful effect of these substances on entomopathogenic fungi and could be an effect of both slight growth inhibition and induction of oxidative stress, which could reduce the infectious potential of the fungi and thus adversely affect their behavior in the environment.

\section{Conclusions}

Entomopathogenic fungi and pyrethroids do not only coexist in the environment but can also be applied together in pest control, and thus it seems highly important to understand the relationship between them. The findings of our study support the hypothesis that pyrethroids influence the metabolism of B. bassiana cells, especially the membrane lipid profile and permeability. By increasing the membrane permeability, pyrethroids gain access into the fungal cells and accumulate within them, leading to the formation of free radicals and reduction of sodium content. Intriguingly, these pesticides were found to cause a decrease in the level of sodium and temporarily lower the amount of the secondary metabolite oosporein, which suggests that their influence on cellular metabolism is very complex. For effective application of these two plant protection agents in pest control, understanding the mechanisms by which pyrethroids affect the metabolism of entomopathogenic fungi is critical, but the obtained results suggest that further research using tools such as proteomics or metabolomics is needed to fully uncover these phenomena.

Received: 23 July 2021; Accepted: 15 October 2021

Published online: 29 October 2021

\section{References}

1. Antwi, F. B. \& Reddy, G. V. P. Toxicological effects of pyrethroids on non-target aquatic insects. Environ. Toxicol. Pharmacol. 40, 915-923 (2015).

2. Cycoń, M. \& Piotrowska-Seget, Z. Pyrethroid-degrading microorganisms and their potential for the bioremediation of contaminated soils: a review. Front. Microbiol. 7, 1-26 (2016).

3. Skolarczyk, J., Pekar, J. \& Nieradko-Iwanicka, B. Zaburzenia immunologiczne wywołane narażeniem na insektycydy z grupy pyretroidów. Postepy Hig. Med. Dosw. 71, 446-453 (2017).

4. Tang, W. et al. Pyrethroid pesticide residues in the global environment: an overview. Chemosphere 191, 990-1007 (2018).

5. Milner, R. J. Selection and characterization of strains of Metarhizium anisopliae for control of soil insects in Australia. In Biological Control of Locusts and Grasshoppers 200-207 (1991).

6. Mascarin, G. M. \& Jaronski, S. T. The production and uses of Beauveria bassiana as a microbial insecticide. World J. Microbiol. Biotechnol. 32, 1-26 (2016).

7. Litwin, A., Nowak, M. \& Różalska, S. Entomopathogenic fungi: unconventional applications. Rev. Environ. Sci. Biotechnol. 19, 23-42 (2020).

8. Forlani, L., Juárez, M. P., Lavarías, S. \& Pedrini, N. Toxicological and biochemical response of the entomopathogenic fungus Beauveria bassiana after exposure to deltamethrin. Pest Manag. Sci. 70, 751-756 (2014).

9. Joshi, M., Gaur, N. \& Pandey, R. Compatibility of entomopathogenic fungi Beauveria bassiana and Metarhizium anisopliae with selective pesticides. J. Entomol. Zool. Stud. 6, 867-872 (2018).

10. Abidin, A. F., Ekowati, N. \& Ratnaningtyas, N. I. Insecticide compatibility to the entomopathogenic fungi Beauveria bassiana and Metarhizium anisopliae. Scr. Biol. 4, 273-279 (2017). 
11. Chen, X.-M., Wang, X.-Y., Lu, W. \& Zheng, X.-L. Use of Beauveria bassiana in combination with commercial insecticides to manage Phauda flammans (Walker) (Lepidoptera: Phaudidae): testing for compatibility and synergy. J. Asia. Pac. Entomol. 24, 272-278 (2021).

12. Tkaczuk, C., Harasimiuk, M., Król, A. \& Beres, P. K. The effect of selected pesticides on the growth of entomopathogenic fungi Hirsutella nodulosa and Beauveria bassiana. J. Ecol. Eng. 16, 177-183 (2015).

13. Feng, M.-G., Johnson, J. B. \& Kish, L. P. Survey of Entomopathogenic fungi naturally infecting cereal aphids (Homoptera: Aphididae) of irrigated grain crops in Southwestern Idaho. Environ. Entomol. 19, 1534-1542 (1990).

14. Puterka, G. J. Fungal pathogens for arthropod pest control in orchard systems: mycoinsecticidal approach for pear psylla control. Biocontrol 44, 183-209 (1999).

15. Xiao, G. et al. Genomic perspectives on the evolution of fungal entomopathogenicity in Beauveria bassiana. Sci. Rep. 2, 59 (2012).

16. Valero-Jiménez, C. A. et al. Comparative genomics of Beauveria bassiana: Uncovering signatures of virulence against mosquitoes. BMC Genom. 17, 1-12 (2016).

17. Li, S., Yi, W., Chen, S. \& Wang, C. Empirical support for the pattern of competitive exclusion between insect parasitic fungi. J. Fungi 7, 189 (2021).

18. Różalska, S., Glińska, S. \& Długoński, J. Metarhizium robertsii morphological flexibility during nonylphenol removal. Int. Biodeterior. Biodegrad. 95, 285-293 (2014).

19. Stolarek, P., Różalska, S. \& Bernat, P. Lipidomic adaptations of the Metarhizium robertsii strain in response to the presence of butyltin compounds. Biochim. Biophys. Acta - Biomembr. 1861, 316-326 (2019).

20. Suchodolski, J., Muraszko, J., Bernat, P. \& Krasowska, A. A crucial role for ergosterol in plasma membrane composition, localisation, and activity of Cdrlp and H+-ATPase in Candida albicans. Microorganisms 7, 1-17 (2019).

21. Nykiel-Szymańska, J., Różalska, S., Bernat, P. \& Słaba, M. Assessment of oxidative stress and phospholipids alterations in chloroacetanilides-degrading Trichoderma spp. Ecotoxicol. Environ. Saf. 1861, 316-326 (2019).

22. de Oliveira, C. N., Neves, P. M. O. J. \& Kawazoe, L. S. Compatibility between the entomopathogenic fungus beauveria bassiana and insecticides used in coffee plantations. Sci. Agric. 60, 663-667 (2003).

23. Kos, K. \& Celar, F. A. Sensitivity of the entomopathogenic fungus Beauveria bassiana (Bals.-Criv.) Vuill. to selected herbicides. Pest. Manag. Sci. 69, 717-721 (2013).

24. Tkaczuk, C., Majchrowska-Safaryan, A. \& Zawadzka, M. The effect of Spinosad and selected synthetic insecticides on the growth of entomopathogenic fungi. J. Res. Appl. Agric. Eng. 58, 194-198 (2013).

25. Soderlund, D. M. Molecular mechanisms of pyrethroid insecticide neurotoxicity: Recent advances. Arch. Toxicol. 86, 165-181 (2012).

26. Liebeskind, B. J., Hillis, D. M. \& Zakon, H. H. Evolution of sodium channels predates the origin of nervous systems in animals. Proc. Natl. Acad. Sci. U. S. A. 108, 9154-9159 (2011).

27. Lee, W. \& Lee, D. G. An antifungal mechanism of curcumin lies in membrane-targeted action within Candida albicans. IUBMB Life 66, 780-785 (2014).

28. Palma-Guerrero, J. et al. Membrane fluidity determines sensitivity of filamentous fungi to chitosan. Mol. Microbiol. 75, 1021-1032 (2010).

29. Chen, Y. et al. Diverse effect of phosphatidylcholine biosynthetic genes on phospholipid homeostasis, cell autophagy and fungal developments in Metarhizium robertsii. Environ. Microbiol. 20, 293-304 (2018).

30. Bernat, P., Gajewska, E., Szewczyk, R., Słaba, M. \& Długoński, J. Tributyltin (TBT) induces oxidative stress and modifies lipid profile in the filamentous fungus Cunninghamella elegans. Environ. Sci. Pollut. Res. 21, 4228-4235 (2014).

31. Czabany, T., Athenstaedt, K. \& Daum, G. Synthesis, storage and degradation of neutral lipids in yeast. Biochim. Biophys. Acta Mol. Cell. Biol. Lipids 1771, 299-309 (2009).

32. Keyhani, N. O. Lipid biology in fungal stress and virulence: entomopathogenic fungi. Fungal Biol. 122, 420-429 (2018).

33. Aouini, F. et al. Short term exposure to permethrin alters lipid classes and fatty acid composition of the unfed clam Donax trunculus from Tunisian coast (Southern Mediterranean). Cah. Biol. Mar. 58, 163-172 (2017).

34. Gao, Q. et al. Phospholipid homeostasis maintains cell polarity, development and virulence in Metarhizium robertsii. Environ. Microbiol. 18, 3976-3990 (2016).

35. Fan, J., Yu, L. \& Xu, C. A central role for triacylglycerol in membrane lipid breakdown, fatty acid $\beta$-oxidation, and plant survival under extended darkness. Plant Physiol. 174, 1517-1530 (2017).

36. Bernat, P., Nykiel-Szymańska, J., Stolarek, P., Słaba, M. \& Różalska, S. 2,4- dichlorophenoxyacetic acid-induced oxidative stress: metabolome and membrane modifications in Umbelopsis isabellina, a herbicide degrader. PLoS One 13, 1002 (2018).

37. Lovett, B. \& St. Leger, R. J. Stress is the rule rather than the exception for Metarhizium. Curr. Genet. 61, 253-261 (2015).

38. Yang, C., Lim, W. \& Song, G. Mediation of oxidative stress toxicity induced by pyrethroid pesticides in fish. Comp. Biochem. Physiol. Part C Toxicol. Pharmacol. 234, 10096 (2020).

39. Kale, M., Rathore, N., John, S. \& Bhatnagar, D. Lipid peroxidative damage on pyrethroid exposure and alterations in antioxidant status in rat erythrocytes: a possible involvement of reactive oxygen species. Toxicol. Lett. 105, 197-205 (1999).

40. Feng, P., Shang, Y., Cen, K. \& Wang, C. Fungal biosynthesis of the bibenzoquinone oosporein to evade insect immunity. Proc. Natl. Acad. Sci. 112, 11365-11370 (2015).

41. Mc Namara, L. et al. Oosporein, an abundant metabolite in Beauveria caledonica, with a feedback induction mechanism and a role in insect virulence. Fungal Biol. 123, 601-610 (2019).

42. Nowak, M., Bernat, P., Mrozińska, J. \& Rózalska, S. Acetamiprid affects destruxins production but its accumulation in Metarhizium sp. spores increases infection ability of fungi. Toxins (Basel). 12, 5903 (2020).

\section{Acknowledgements}

The research was financed from the grant of the National Science Center of Poland under the number 2016/23/B/ NZ9/00840.

\section{Author contributions}

Conceptualization (S.R.), methodology (A.L., P.B, M.N., M.S), validation (A.L., P.B, M.N., M.S), investigation (A.L., P.B, M.N., M.S), writing (S.R., A.L.), supervision (S.R.), founding acquisition and project administration (S.R.).

\section{Competing interests}

The authors declare no competing interests.

\section{Additional information}

Supplementary Information The online version contains supplementary material available at https://doi.org/ 10.1038/s41598-021-00702-y. 
Correspondence and requests for materials should be addressed to S.R.

Reprints and permissions information is available at www.nature.com/reprints.

Publisher's note Springer Nature remains neutral with regard to jurisdictional claims in published maps and institutional affiliations.

(c) (i) Open Access This article is licensed under a Creative Commons Attribution 4.0 International cc) License, which permits use, sharing, adaptation, distribution and reproduction in any medium or format, as long as you give appropriate credit to the original author(s) and the source, provide a link to the Creative Commons licence, and indicate if changes were made. The images or other third party material in this article are included in the article's Creative Commons licence, unless indicated otherwise in a credit line to the material. If material is not included in the article's Creative Commons licence and your intended use is not permitted by statutory regulation or exceeds the permitted use, you will need to obtain permission directly from the copyright holder. To view a copy of this licence, visit http://creativecommons.org/licenses/by/4.0/.

(C) The Author(s) 2021 